\title{
Auf dem Weg zur Kammer
}

\begin{abstract}
Nach dem erfolgreichen Start der ersten Landespflegekammer in Rheinland-Pfalz sind in Schleswig-Holstein und Niedersachsen Pflegekammern im Aufbau. Wir fragten Patricia Drube und Iris MeyenburgAltwarg: Wie ist der Stand der Dinge in Ihren Bundesländern?
\end{abstract}

? Wie weit sind Sie mit den Vorbereitungen zur Pflegekammer?

Drube: In Schleswig-Holstein hat der Errichtungsausschuss, der aus 26 engagierten Pflegefachpersonen aller drei Berufsgruppen besteht, im Januar seine Arbeit aufgenommen. Dass davon öffentlich bisher nicht allzu viel wahrnehmbar ist, liegt daran, dass wir erst einmal arbeitsfähig werden mussten. Aufgaben verteilen, Termine finden oder vereinbaren, wie Entscheidungen getroffen werden. Inzwischen haben wir eine Hauptsatzung, eine Geschäftsordnung und eine Entschädigungsordnung. Seit 1. Juni haben wir unsere Geschäftsstelle in Neumünster. Seit dem 1. Juli beschäftigen wir dort unsere erste hauptamtliche Mitarbeiterin. Der Auftrag für die Gestaltung unserer Internetseite und die Begleitung unserer Informationskampagne ist vergeben. Und: Spätestens am 9. Juni 2018 muss die Kammerversammlung gewählt sein.

Meyenburg-Altwarg: Am 28. Juli 2015 fand in Niedersachsen die konstituierende Sitzung der Gründungskonferenz mit 25 ordentlichen Mitgliedern und 25 Vertretungen aus allen Berufsfeldern der Pflege statt. Alle Mitglieder haben eine pflegerische Grundausbildung und mehrere Jahre Berufserfahrung. In vier zentralen Arbeitsgruppen - Kommunikation, Infrastruktur, Satzung und Registrierung - beschäftigen wir uns einmal im Monat ganztägig mit einzelnen Aufgabenpaketen der Gründungskonferenz, zusätzlich geschieht dies in Arbeits- und Arbeitsuntergruppen.

Aktuell liegen wir sehr gut im Zeitplan. Wir haben ein Organisationskonzept für die Kammer, inklusive Finanzplanung, Räumlichkeiten, benötigte Fachkompetenz und IT-Versorgung vorbereitet und die Beschlussvorlagen für die administrativen Vorgänge ausgearbeitet. Diese werden der kommenden Errichtungskonferenz zur Verfügung gestellt. Konkret warten wir jetzt auf die Verabschiedung des Gesetzes über die Pflegekammer Niedersachsen, der Entwurf wurde in den Landtag eingebracht.

? Welches sind derzeit die wichtigsten Aufgaben des Errichtungsausschusses? Drube: Unser Kernauftrag ist, alle in Schleswig-Holstein tätigen Berufsangehörigen zu registrieren und bis spätestens Juni 2018 die Kammerwahl durchzuführen. Der erste Schritt ist, mit einer breit angelegten Kampagne darüber zu informieren und natürlich auch über den Sinn und Zweck der Kammer. Dabei geht es auch darum, die Kolleginnen und Kollegen zu motivieren, sich aktiv einzubringen und für die Wahl zu kandidieren.

Meyenburg-Altwarg: Um dem Ziel einer berufsständische Selbstverwaltung näher zu kommen, ist die Vermittlung von Informationen an die Pflegefachpersonen vor Ort sehr wichtig. In den letzten Monaten haben wir mehr als 150 Veranstaltungen in ganz Niedersachsen angeboten. Wichtig war es uns, die Pflegenden selbst, aber auch andere Interessierte über den aktuellen Sachstand zu informieren. Außerdem wollten wir auf die Chancen der Pflege zur Selbstbestimmung durch die Kammer aufmerksam machen und Fragen beantworten. Im nächsten Schritt geht es darum, nach der Gesetzesverabschiedung die Tätigkeit im Errichtungsausschuss aufzunehmen und dann insbesondere die Themen "Registrierung" und "Kammerwahl" vorzubereiten. Wir gehen davon aus, dass der Start der Pflegekammer Anfang 2018 sein wird.

? Erfolgreich gestartet ist vor über 100 Tagen die Landespflegekammer Rhein-

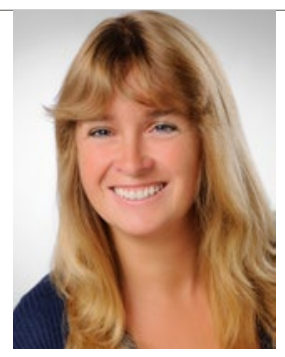

Patricia Drube

Vorsitzende des Errichtungsausschusses der Pflegekammer SchleswigHolstein

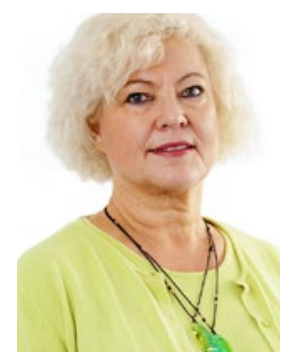

Iris MeyenburgAltwarg

Vorsitzende der Gründungskonferenz zur Pflegekammer Niedersachsen

land-Pfalz. Was bedeutet dies für die Situation in Ihrem Bundesland?

Drube: Die Kolleginnen und Kollegen aus Rheinland-Pfalz unterstützen und beraten uns, wo immer das möglich ist. Auch die Ministerien stehen in gutem Kontakt. Das hilft uns sehr. Allerdings machen wir auch die Erfahrung, dass es viele Unterschiede in den gesetzlichen Grundlagen gibt und man nicht alles auf Schleswig-Holstein übertragen kann. In jedem Fall profitieren wir von der öffentlichen Aufmerksamkeit, die durch die Kammergründung in Rheinland-Pfalz entstanden ist.

Meyenburg-Altwarg: Auch uns hat die Pflegekammer in Rheinland-Pfalz während der gesamten Phase der Gründungskonferenz in hohem Maße unterstützt. Durch die sehr offene und kollegiale $\mathrm{Zu}$ sammenarbeit ist es gelungen, in Niedersachsen in kurzer Zeit eine klare Struktur und einen geeigneten Arbeitsmodus für die Planung und Vorbereitung der Kammer zu entwickeln.

Das Interview führte Ute Burtke 